\title{
Design of a Sprout Layer-rack Aeroponic Cultivation Device GUO $\mathrm{Yi}^{1, \mathrm{a}}$, CHEN Lanfen ${ }^{2, \mathrm{~b}}$, ZHANG Guiqin ${ }^{3, \mathrm{c}}$
}

\author{
${ }^{1}$ Department of Horticulture Beijing Vocational College of Agriculture, Beijing, 102442, China \\ ${ }^{2}$ Training Management Center, Beijing Vocational College of Agriculture, Beijing, 102442, China \\ ${ }^{3}$ Beijing Green Valley Sprouts Co., Ltd, Beijing, 100074, China \\ aemail:guoyibvca@163.com, bemail:372090241@qq.com, cemail:zhangguiqin0405@sina.com
}

Keywords: sprout, layer-rack, aeroponic cultivation device, design

\begin{abstract}
. a sprout layer-rack aeroponic cultivation device has been designed to solve the problems of low space utilization rate, large consumption of irrigation water, unbalanced growth amount and low automaticity in sprout production. SolidWorks has been employed to build 3D models for spare parts and the overall structure. Application tests have indicated that sprouts cultivated with this system grow well and produce basically the same growth amount in the upper and the lower layers. Compared with the traditional flat cultivation, the vertical cultivation can increase space utilization ratio by $300 \%$. Compared with the traditional spray mode, the aeroponics cultivation mode can produce booming sprouts, with the root length increased by more than $30 \%$, yield more than $20 \%$ and irrigation water saved by more than $40 \%$. This system has been reasonably designed, reliably applied and easily used. After adjustment of the structural dimensions, this system can be applied to cultivation of spouts of different scales and thus shows great application prospect.
\end{abstract}

\section{Introduction}

Along with growth in the living standard and change in people's food habits, people are no longer satisfied with the supply quantity of vegetables, but pay more attention to the appearance, quality and safety of such vegetables. Sprouts have earned consumers' love with their rich nutrition and no pollution. Sprouts feature a short production cycle, high efficiency, less investment, fast effect and handsome earnings. They can be produced intensively in production plants and greenhouses, or cultivated on the balcony [1] [2].

Aeroponics cultivation is a kind of soilless culture method, under which, the root system of the plants grows in a closed and light-tight aeroponics space to receive the maximized supply of oxygen and grow under fine conditions. In this condition, vegetables can grow fast [3] [4]. Compared with traditional soil culture, this method can save substantive water and thus makes an advanced cultivated model in modern agricultural production [5] [6].

Survey on sprout producers shows that they mainly adopt flat cultivation or vertical cultivation, and the sprinkling irrigation, featuring low automaticity. Flat cultivation has good light conditions and balanced growth amount, but lower space utilization ratio. By contrast, the vertical cultivation features higher space utilization ratio, but poorer light conditions in the lower layers and unbalanced growth amount. The sprinkling irrigation method causes high humidity in the space, which makes sprouts vulnerable to diseases and leads to severe waste of water resources. In this paper, a sprout layer-rack aeroponic cultivation device has been designed to effectively solve problems stated above.

\section{Overall Structure and Working Process of the Sprout Layer-rack Aeroponic Cultivation Device}

The device is composed of the layer-rack structure, cultivation system, spray system, supplemental lighting system and control system. The layer-rack system is used to support the cultivation system and install the supplemental lighting system and control system. The cultivation system is used for cultivation of sprouts and the spray system for producing gas spray for aeroponics cultivation of 
sprouts. The control system is designed for opening and closing the spray system and the supplemental system at a fixed time. During operation, the control system opens and closes the supplemental lighting system and spray system at the set time. The working process is shown in Fig. 1.

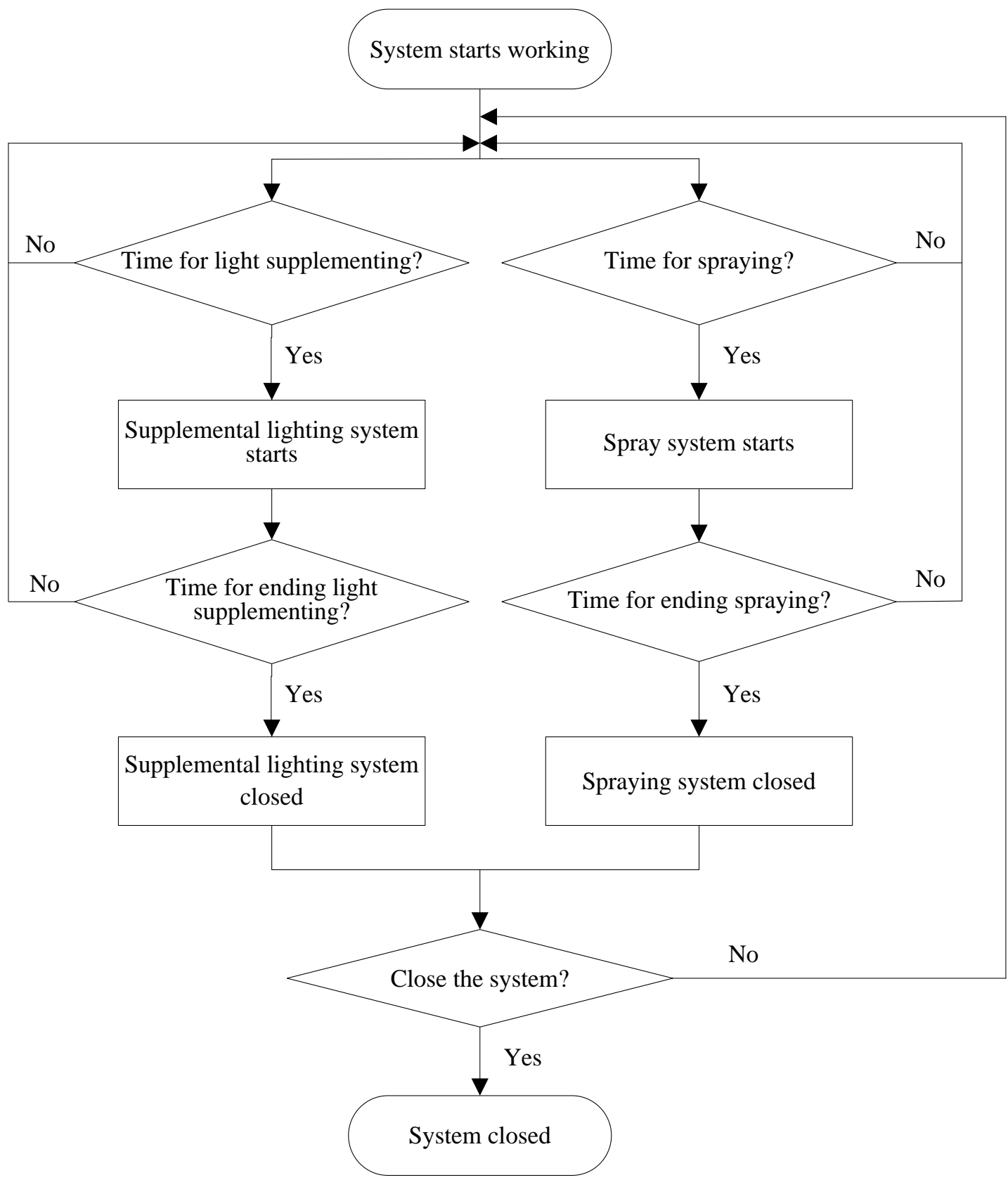

Fig. 1 Working process

\section{Design of the Sprout Layer-rack Aeroponic Cultivation Device}

Design Method. SolidWorks software, a Windows-based 3D CAD system, is capable of providing different design solutions, reducing errors in the design process and improving product quality and design efficiency [7] [8]. According to the overall structure of the device, SolidWorks is employed as the design tool [9] [10] [11]. The design process is first to design the cultivation system, then the layer rack structure according to the design parameters of the cultivation system, the design of spare parts for the spray system, supplemental lighting system and the control system, and finally the sprout layer-rack aeroponic cultivation device.

Design of the Cultivation System. The cultivation system is consisted of the seedling tray and the cultivation tank. The seedling tray adopts the universal flat-bottom multihole sprout seedling tray, being $600 \mathrm{~mm} * 230 \mathrm{~mm} * 35 \mathrm{~mm}$ in this case. The cultivation tank is welded with the stainless 
steel plates, with the upper part of the cultivation tank to place the seedling tray. According to the cultivation scale, each cultivation tank can hold one or more seedling trays. In this design, each cultivation tank can hold four seedling trays. On one side of the cultivation tank are two through holes, one for installing spray pipelines and the other for installing the return water pipelines. The cultivation system is shown in Fig. 2.

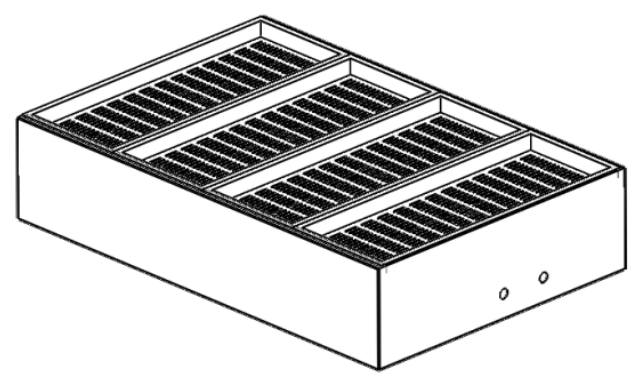

Fig. 2 Cultivation system

Design of the Layer-rack Structure. The layer-rack structure has been made of universal angle steel to facilitate assembly and disassembly. It is composed of the upright, tank beam and the side beam. To increase the space utilization ratio, the structure has been designed into many layers, three layers in this case with interval of $40 \mathrm{~mm}$, according to the cultivation scale. To ensure structure stabilization of each joint, the triangular plate and bolting have been applied at each joint. The design parameters of the layer-rack structure have been determined according to the design parameters of the cultivation system. In this design, the layer-rack structure has been designed to be 930mm (L)*630mm (W)*1500mm (H). The layer-rack structure is shown in Fig. 3.

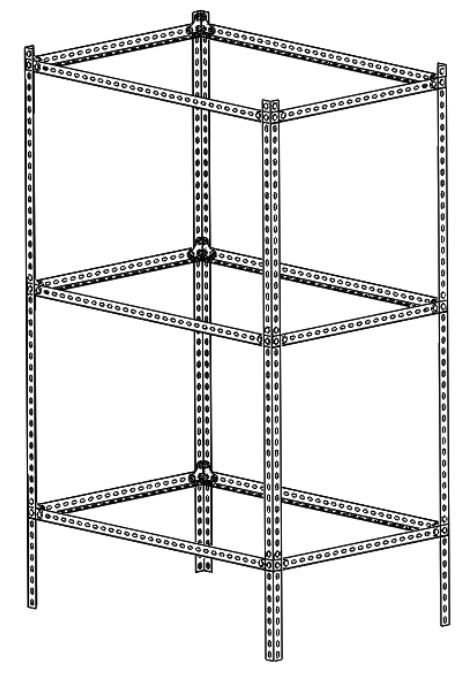

Fig. 3 Layer-rack structure

Design Of The Spray System. The spray system is consisted of the water tank, water pump, filter, spray pipeline, spray nozzle and return water pipeline. The water tank adopts PP boards adhesively bonded with each other, for holding water for spraying. The water tank is installed on the lower part of the layer-rack structure. The water pump pressurizes water. In this design, the $100 \mathrm{~W}$ high pressure diaphragm pump is used, with working pressure $7 \mathrm{Kg}$ and flow $8 \mathrm{~L} / \mathrm{min}$. The filter is used to filter impurities in the spraying water; in this design, the laminated filter is applied. The spray pipeline adopts the $16 \mathrm{~mm}$ PE pipes. The spraying nozzle is used to spray water; in this design, the microsprinkling nozzle with diameter $0.5 \mathrm{~mm}$ is selected. The return water pipeline is used for holding the back flow of water left after being sprayed; in this design, the $16 \mathrm{~mm}$ PVC pipe is used. The spray system is shown in Fig. 4.

Design of the Supplemental Lighting System. The supplemental lighting system is consisted of the supplemental lighting lamp and the wire. For lack of lighting in the lower layers, the sprout multilayer vertical cultivation rack needs artificial light source for supplemental lighting. In this 
design, the LED plant growth light has been adopted, with red light 630-660nm and blue light 450-470nm (ratio between red light and blue light is 2:1).

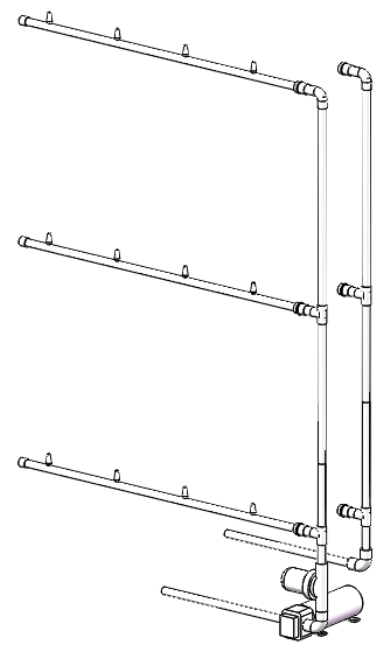

Fig. 4 Spray system

Design of the Control System. The control system is composed of the control box, SCM system and the power supply adapter. The control box is used for installing SCM system and the power supply adapter. The SCM system is used to control time opening and closing of the spray system and the supplemental lighting system. The power supply adapter is used for power supply of the feed pump and the supplemental lighting lamp. This system enables automatic spraying and supplemental lighting in growth process of the sprouts.

Design of the Sprout Layer-rack Aeroponic Cultivation Device. After all spare parts have been designed, SolidWorks is employed to realize virtual assembly according to connection relationships between spare parts and through adding constraints and perform interference check with its interference check function. After that, according to the check results, the design of spare parts will be consummated [12] to finally produce the design of the sprout layer-rack aeroponic cultivation device, as shown in Fig. 5.

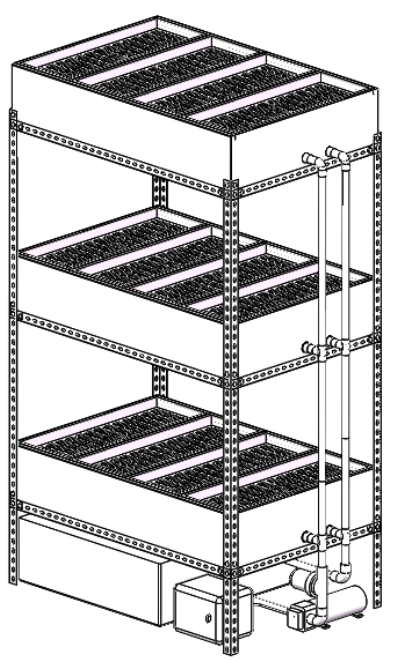

Fig. 5 Sprout layer-rack aeroponic cultivation device

\section{Application of the Sprout Layer-rack Aeroponic Cultivation Device}

Utilizing the design results, the sprout layer-rack aeroponic cultivation device has been produced and applied on aeroponics cultivation of pease, buckwheat, oil sunflower, wheat and radish in the sunlight greenhouse of Beijing Vocational College of Agriculture Practical Training Management Center. Results show that sprouts cultivated with this system grow well and produce basically the 
same growth amount in the upper and the lower layers. Compared with the traditional flat cultivation, the vertical cultivation can increase space utilization ratio by $300 \%$. Compared with the traditional spray mode, the aeroponics cultivation mode can produce booming sprouts, with the root length increased by more than $30 \%$, yield more than $20 \%$ and irrigation water saved by more than $40 \%$.

\section{Conclusion}

Application results of this device show that this system has been reasonably designed, reliably applied and easily used. It can evidently enhance the cultivation space utilization ratio, improve the yield and save irrigation water significantly. The physical dimension of this system is subject to change according to the production scale to adapt to diversified cultivation scales. Thus it can be used in cultivation of sprouts grown on the balcony, grown in a small scale or grown in a large scale in plant factories.

\section{Acknowledgements}

This paper is co-funded by Test and Demonstration of Efficient Production Model of Sprouts project (Project No. 20150128) and Water-saving Irrigation Technology in the Production of Sprouts project (Project No. 20150203-13) under Beijing Municipal Commission of Rural Affairs

\section{References}

[1] Tan Zizhen, Li Qingzhou. Disquisition on Application Feasibility of Seedling Vegetable Planting on High Seas Vessels [J]. Journal of Anhui Agricultural Sciences, 2015, 43(2): 391-392.

[2] Long Dongling, Zhu Qiujin, Zhou Wenmei, Wei Kezhou. Different Treatment on the Influence of Growth and Yield of Perilla Frulescens Sprouts [J]. Journal of Guizhou University (Natural Sciences), 2016, 33(3): 35-38.

[3] Gao Guohua, Wang Tianbao. Design of Aeroponics Greenhouse Vegetable Harvester Harvesting Mechanism [J]. Journal of Agricultural Mechanization Research, 2015(10): 91-97.

[4] Ling Min, He Chunmei, Gao Jianmin, Wang Hongfeng. Effects of Aeroponics on the Growth, Yield and Quality of Plantago asiatica [J]. Guangdong Forestry Science and Technology, 2015(1): 29-33.

[5] Wang Shibin, Li Baohai, Zhu Rongjie, Yang Bin. Study on Growing Development of Eight Leaf Vegetables with Aeroponical Culture in Greenhouse [J]. Southwest China Journal of Agricultural Sciences, 2015, 28(4): 1854-1856.

[6] Wen Jing, Cheng Ruifeng, Meng Lili, Wei Jinhe, Yang Qichang, Zhang Jun. Development and Application Effect of Ultrasonic Aeroponic Cultivation Device [J]. Acta Agriculture Jiangxi, 2012, 24( 1): 23-25.

[7] Zhao Qinglai, Yuan Jun, Wang Jingli. Digital design and simulation of variable force seedling belt roller [J]. Journal of Chinese Agricultural Mechanization, 2015, 36(5): 58-59, 63.

[8] Qin Weihua, Wang Longfei, Wang Wanzhang. Virtual Prototype Design and Research of Tobacco Rotary Seedling Machine [J]. Journal of Agricultural Mechanization Research, 2014(1): 144-147.

[9] Pan Shiqiang, Cao Zifu, Zhao Wanning, Yang Yulin. Design on corn straw bundling machine based on SolidWorks [J]. Journal of Chinese Agricultural Mechanization, 2016, 37(6): 31-34.

[10] Liao Jinwei, Wu Weibin, Feng Yunlin, Xu Pengbo. Design and Simulation of Hydraulic Retarder Based on the Mountain Orchard Transporter [J]. Journal of Agricultural Mechanization 
Research, 2016(8): 82-86.

[11] Guo Xiaohuan, Dai Fei, Zhao Wuyun, Shi Linrong, Shuai Zhongkui, Wu Yifei. Design of the whole double furrow filmpicking baler machine [J]. Journal of Chinese Agricultural Mechanization, 2016, 37(9): 32-36, 55.

[12] Peng Tian-hao, Wang Guang-yao, Zhang Yi-long, Huang Peng, Zhong Wei-cai. Design of shearer simulated spiral drum based on similarity theory [J]. Chinese Journal of Engineering Design, 2016, 23(4): 322-326. 